\title{
Cooperative Communication for Multi-Hop Routing in ZigBee Network
}

\author{
Chang-Bin Ha, Hyoung-Kyu Song* \\ Ut Communication Research Institute, Sejong University, Seoul, Korea (E-mail: zhkdldj@naver.com) \\ Ut Communication Research Institute, Sejong University, Seoul, Korea (E-mail: songhk@sejong.ac.kr)
}

\begin{abstract}
In this paper, we propose multi-hop routing scheme using RSSI to improve the reliability of ZigBee network. By using RSSI for the routing, the transmitted signal is less affected by the fading in each link on the routing path. If the proposed routing scheme is applied to relaying, the demodulation error is reduced in each relay node and the performance is significantly improved as the number of hops increases. Also, if the proposed scheme is applied to the cooperative communication, the improved diversity gain is additionally obtained.
\end{abstract}

Keywords - ZigBee, multi-hop routing, RSSI

\section{INTRODUCTION}

Wireless LAN, which is a communication field that can process high-speed data, has a problem that it is expensive and consumes a large amount of power to be applied to the fields of home network, build automation, industrial/commercial automation, health care and control system. Because the above mentioned areas can achieve their purpose through low-speed data transmission technology, a low-cost, low-power consumption communication technology is required. To meet these requirements, the ZigBee alliance enacted ZigBee in 2006 [1]. Since the ZigBee specification has been enacted, the commercialization efforts in various fields such as ZigBee transceiver, network stack, and ZigBee application system are actively being made. ZigBee features long battery life, network self-configuration, reliable communication, network scalability and operational availability [2]-[4].

The network layer of ZigBee defines the required functions for network operation such as network configuration, node discovery procedure, address assignment method, hierarchical routing, and mesh routing method based on routing table [5][6]. Especially, the hierarchical routing is a suitable routing method for device with very low memory resource. In the hierarchical routing, each node does not use the routing table and selects the own child node or parent node as an intermediate node on the routing path. However, since links to neighboring nodes other than the child and parent nodes are not considered, there is a problem of inefficiency for a routing cost.

In this paper, to solve the problem of inefficiency for routing, the method selecting the neighboring node as the intermediate node that can reduce the routing cost is proposed.

\section{SySTEM MODEL}

Fig. 1 shows the system model of ZigBee network. ZigBee network consists of a full function device (FFD) and reduced function device (RFD). FFD can participate in the network with three modes of the coordinator, router, and end device. Also, there is no constraint for the configuration of network topology and it is possible to communicate with all nodes on the network. On the other hand, RFD can participate in the network with only end device mode. Also, RFD is configurable for only limited star topology and can communicate with only the parent router. The reason for the devices participating in the network is divided by FFD and RFD is to minimize power consumption by minimizing the function of RFD. Because the routers are intertwined with multiple paths, even if some of the routers become inoperable, ZigBee can find alternate routes. 


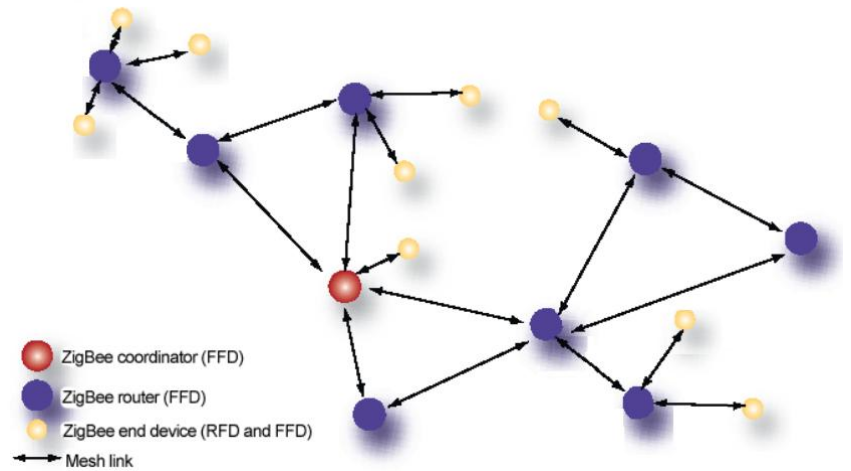

Fig. 1: ZigBee mesh network consisting of FFD and RFD

\section{Proposed Multi-Hop Routing COMMUNICATION}

The received signal of relay node is represented as follows,

$$
y_{s, r}=\sqrt{P} h_{s, r} x+n_{s, r}, \quad(1)
$$

where $\sqrt{P}$ is the transmission power and $h_{s, r}$ is the channel between the source and relay node. Also, $x$ is the transmitted signal and $n_{s, r}$ is the additive white Gaussian noise (AWGN) in the relay node. The received signal of destination node is represented as follows,

$$
y_{s, d}=\sqrt{P} h_{s, d} x+n_{s, d},
$$

where $h_{s, d}$ is the channel between the source and destination node and $n_{s, d}$ is AWGN in the destination node. The equations can be extended to multi-hop.

The cooperative communication can be classified into the amplify and forward (AF) and decode and forward (DF). In the AF-based cooperative communication, the relay node amplifies the received signal and transmits the amplified signal to the destination node. The amplification factor is as follows,

$$
\beta_{r}=\frac{\sqrt{P}}{\sqrt{P\left|h_{s, r}\right|^{2}+N_{0}}},
$$

where $N_{0}$ is power of noise. The received signal of destination node through the relay node is as follows,

$$
y_{r, d}=\beta_{r} \sqrt{P} h_{r, d} h_{s, r} x+n_{r, d},
$$

where $h_{r, d}$ is the channel between the relay and destination node and $n_{r, d}$ is AWGN in the destination node. The combined received signal is as follows,

$$
y=a_{1} y_{s, d}+a_{2} y_{r, d}
$$

where $a_{1}$ and $a_{2}$ are as follows,

$$
\begin{gathered}
a_{1}=\frac{\sqrt{P} h_{s, d}^{*}}{N_{0}}, \quad(6) \\
a_{2}=\frac{\sqrt{\frac{P}{P\left|h_{s, r}\right|^{2}+N_{0}}} \sqrt{P} h_{s, r}^{*} h_{r, d}^{*}}{\left(\sqrt{\frac{P\left|h_{r, d}\right|^{2}}{P\left|h_{s, r}\right|^{2}+N_{0}}}+1\right) N_{0}},
\end{gathered}
$$

$a_{1}$ and $a_{2}$ are designed to maximize signal to noise ratio (SNR) of the combined signal. In the DF-based cooperative communication, the relay node decodes the received signal and transmits the re-modulated signal to the destination node. The received signal of destination node through the relay node is as follows,

$$
y_{r, d}=\sqrt{P_{2}} h_{r, d} \hat{x}+n_{r, d},
$$

where $\sqrt{P_{2}}$ is the transmission power of relay node and $\hat{x}$ is re-modulated signal. In the DF-based cooperative communication, the combining factors are as follows,

$$
\begin{gathered}
a_{1}=\sqrt{P} h_{s, d}^{*} / N_{0}, \quad(9) \\
a_{2}=\sqrt{P_{2}} h_{r, d}^{*} / N_{0} .
\end{gathered}
$$

The link between each node is determined by the received signal strength indicator (RSSI). If a single relay node is used for routing, the relay node is determined in according to RSSI in the relay node. Also, if multiple relay nodes are used for routing, the relay nodes are determined in according to the average of RSSI in each relaying link.

\section{Simulation Results}

In the multi-hop communication, the reliability is significantly reduced by the demodulation error in each node on the routing path. The demodulation error occurs with a higher probability as the fading between links becomes worse. Therefore, the simulation is performed in according to the distance that most directly affects 
the fading. Also, simulation is performed on $1 \sim 3$ multi-hop environment over the LOS and NLOS channel models.

Fig. 2, 3, and 4 show the simulation results in the relaying and cooperative communication based on $\mathrm{AF}$ and $\mathrm{DF}$, respectively. Because the demodulation error can occur in all relay nodes on the route, the proposed scheme has significantly improved performance as the number of hops increases. Also, the application of the proposed technique for cooperative communication can improve diversity gain by improving the fading effect of the additional link.

\section{Conclusion}

In ZigBee network, the routing algorithm that transmits data to efficient route is very important. Also, the routing algorithm with highcomplexity is not suitable for ZigBee routing. The proposed method uses RSSI as a routing cost and transmits data to a destination node through links for neighboring nodes. Because RSSI directly represents the channel state between each node, it is the most intuitive routing cost for the reliability of communication. The simulation is performed by applying the proposed routing algorithm to the relaying, $\mathrm{AF}$, and $\mathrm{DF}$ techniques. The simulation shows that the communication reliability of ZigBee network is significantly improved by the proposed scheme.

\section{ACKNOWLEDGEMENTS}

This work was supported by the Korea Institute of Energy Technology Evaluation and Planning(KETEP) and the Ministry of Trade, Industry \& Energy(MOTIE) of the Republic of Korea (No. 20161210200670) and was supported by the IT R\&D program of MOTIE/KEIT [10054819, Development of modular wearable platform technology for the disaster and industrial site]. (Corresponding Author: Hyoung-Kyu Song)

\section{REFERENCES}

[1] IEEE Std 802.15.4-2006. Wireless Medium Access Control (MAC) and Physical Layer (PHY) Specifications for Low-Rate Wireless Personal Area Networks (WPANs).

[2] C. Gomez, J. Paradells, Wireless Home Automation Networks: A Survey of Architectures and Technologies, IEEE Communication Magazine, 48(6), 2010, 92 101.

[3] H. A. Keshavarz, V. Ribeiro, and R. Riedi, Broadcast Capacity in Multihop Wireless Networks, Proceedings of the $12^{\text {th }}$ Annual International Conference on Mobile Computing and Networking, 2006, 239-250.
[4] R. Zheng, Information Dissemination in Power-constrained Wireless Networks, Proceedings of the $25^{\text {th }}$ IEEE International Conference on Computer Communications, 2006, 1-10.

[5] Z. Bidai, H. Haffaf, and M. Maimour, Node Disjoint Multi-Path Routing for ZigBee Cluster-Tree Wireless Sensor Networks, Multimedia Computing and Systems, 2011, 1-6.

[6] Z. Bidai, M. Maimour, Multipath Routing for High-Data Rate Applications in ZigBee Wireless Sensor Networks, New Technologies, Mobility and Security, 2014, $1-5$. 

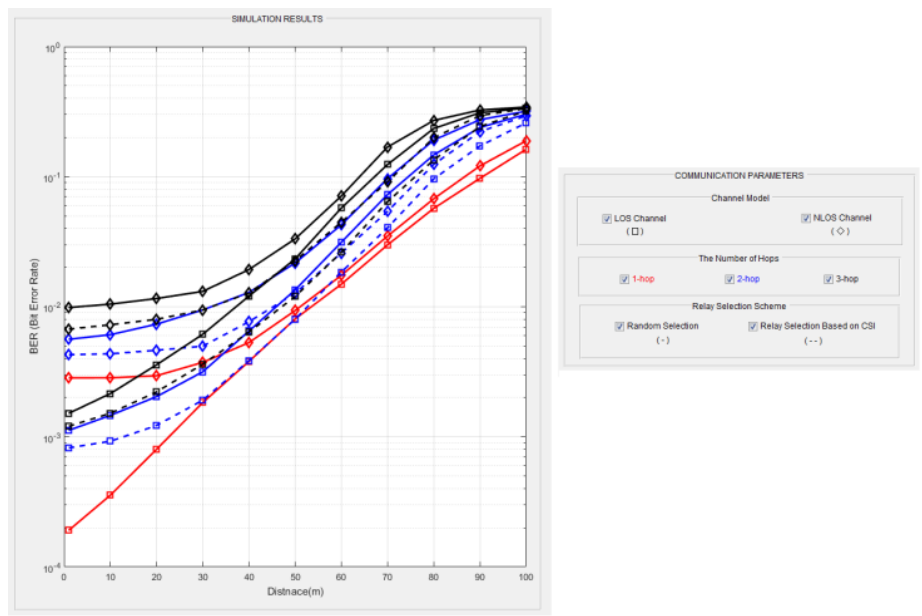

Fig 2: Comparison of proposed and conventional scheme in relaying communication

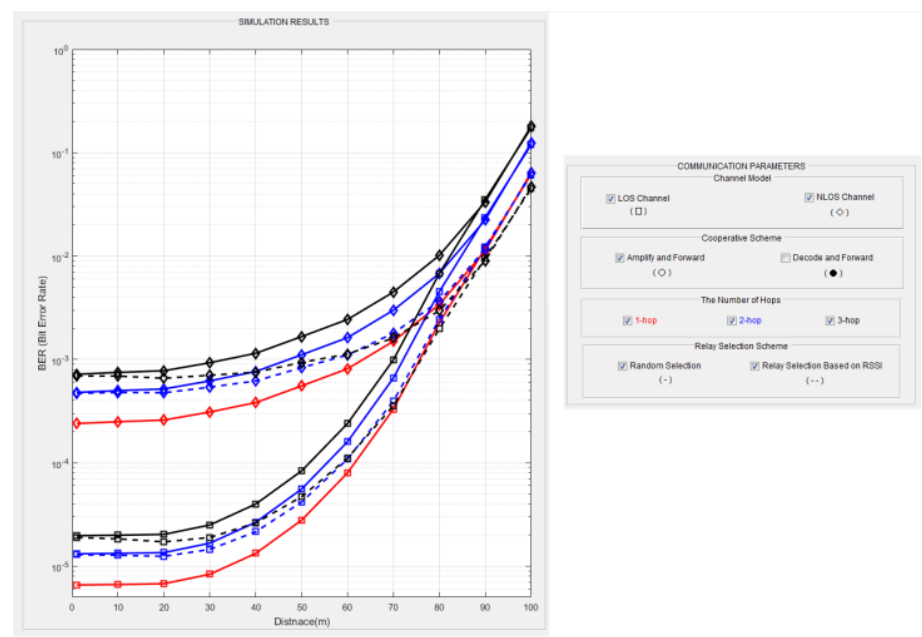

Fig 3: Comparison of proposed and conventional scheme in the cooperative communication based on AF

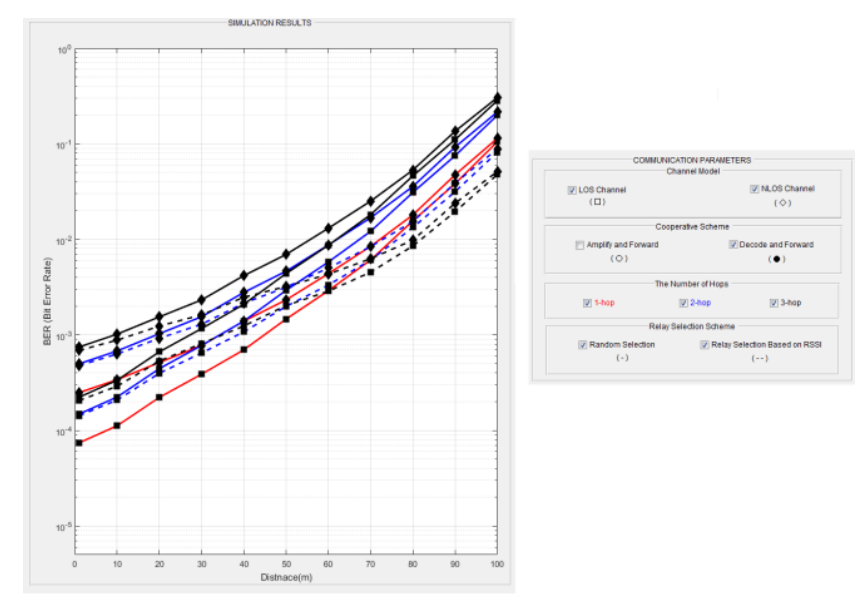

Fig 4: Comparison of proposed and conventional scheme in the cooperative communication based on DF 